\title{
MRI of uncommon and unusual lesions of the duodenum, small bowel, and mesentery
}

\author{
James F. Glockner, Christine U. Lee \\ Mayo Clinic, Rochester, MN, USA. \\ Correspondence: Christine U Lee. Address: Mayo Clinic 200 First St, SW Rochester, MN 55905, USA. \\ Email: lee.christine@mayo.edu \\ Received: January 26, 2015 \\ Accepted: March 23, $2015 \quad$ Online Published: April 7, 2015 \\ DOI : $10.5430 /$ ijdi.v2n2p43 \\ URL: http://dx.doi.org/10.5430/ijdi.v2n2p43
}

\begin{abstract}
As MRI assessment of bowel increases, unusual and uncommon lesions of small bowel and mesentery are more frequently encountered. MRI is often useful for characterizing these lesions, and sometimes a specific diagnosis can be made. This manuscript illustrates and discusses the MRI appearance of a variety of unusual lesions involving or related to the small bowel and mesentery.
\end{abstract}

\section{Keywords}

MRI, Duodenum, Small bowel, Mesentery, MR enterography

\section{I ntroduction}

Body MRI has long been considered only marginally useful for identifying and characterizing pathology of large and small bowel in comparison to endoscopy, conventional fluoroscopy, CT enterography, and capsule endoscopy. This perception has changed with the development of MR enterography (MRE), which has proven highly effective for detection and characterization of inflammatory bowel disease (IBD) ${ }^{[1-3]}$.

MRE represents one of the most rapidly growing segments of many body MRI practices, and the increasing caseload (as well as corresponding increased scrutiny of bowel) has led to the detection of several unusual lesions involving small bowel and mesentery. An unusual lesion found during the course of an MRE examination to some extent represents the ideal scenario, where bowel has already been distended with oral contrast, and motion artifact reduced with the use of an antiperistaltic agent. Even when such a lesion is serendipitously encountered during an abdominal MRI focused on the liver, pancreas, or kidneys, it is still often possible to obtain images of acceptable quality.

Although a precise diagnosis may not always be possible for the unusual small bowel or mesenteric mass, MRI offers many opportunities for lesion characterization, and the range of possibilities can often be narrowed with a well-performed examination. This manuscript briefly discusses methods for optimizing bowel imaging with MRI and illustrates the MRI appearance of a variety of unusual lesions involving or related to the small bowel and mesentery. 


\section{Technical considerations}

MRE protocols are primarily designed to optimize small bowel visualization in the setting of inflammatory bowel disease. Important considerations in developing MRE protocols include 1) adequate distention of small bowel (and large bowel if possible) - our patients ingest approximately 1.5 liters of an oral contrast agent containing sorbitol beginning approximately one hour prior to their examination; 2) reduction of peristalsis - our protocol includes intravenous injection of an antiperistaltic agent (glucagon) just before gadolinium is administered. MR enterography protocols should emphasize fast T2-weighted sequences (SSFSE and b-SSFP) and dynamic gadolinium-enhanced 3D SPGR acquisitions in coronal and axial planes.

Balanced steady state free precession (b-SSFP) pulse sequences provide T2/T1 contrast weighting, and 2D images can be obtained with rapid sequential acquisition, so that antiperistaltic agents are not required, and the acquisition time per image is typically $<1$ second. Despite their short TR and TE values, b-SSFP images generally have high signal to noise ratios (SNR), and overlapping slices can be obtained without significant signal loss. Single shot fast spin echo (SSFSE) pulse sequences are T2-weighted images that can also be obtained with rapid sequential acquisition, thereby minimizing motion artifact from peristalsis. SSFSE images are generally somewhat less artifact-prone than b-SSFP images; however when the effective TR is short (to minimize the acquisition time per slice) or when overlapping slices are acquired, saturation effects may cause signal loss.

Dynamic gadolinium-enhanced 3D SGPR images are typically performed in the coronal plane, with arterial, portal venous, and equilibrium phase acquisitions, followed immediately by axial 3D SPGR images. These images should be acquired after administration of antiperistaltic agents. Post-gadolinium images can be limited by motion artifact from peristalsis or inadequate breath-holding, and therefore the spatial resolution of each acquisition should be adjusted according to the breath-holding capacity of the individual patient.

Lesions involving bowel or mesentery may occasionally be serendipitously encountered on examination not dedicated to bowel imaging. In these cases, additional SSFSE and/or b-SSFP acquisitions in planes oriented parallel and perpendicular to the mass or to the associated bowel loop may be helpful in providing motion-free images of the lesion. Diffusionweighted images may also be useful - these should generally be obtained with a relatively high b-value $\left(>800 \mathrm{~s} / \mathrm{mm}^{2}\right)$ so that the $\mathrm{T} 2$ shine-through effects from fluid within the bowel lumen are reduced.

\subsection{Duodenal lesions}

Peptic ulcer disease (PUD) (see Figure 1) is a major cause of morbidity and mortality. The pathogenesis of most ulcers is related to Helicobacter pylori infection and/or the use of nonsteroidal anti-inflammatory medications ${ }^{[4]}$. The duodenum is the $2^{\text {nd }}$ most common location for PUD following the stomach, accounting for $38 \%$ of ulcers ${ }^{[5]}$.

Figure 1. Axial post-gadolinium 3D SPGR image from a 56 year-old man with upper abdominal pain demonstrates a large ulcer (arrows) along the lateral margin of the $2^{\text {nd }}$ portion of the duodenum. Note also mild mural thickening and enhancement along the neck of the ulcer (arrows). Findings were confirmed at endoscopy.

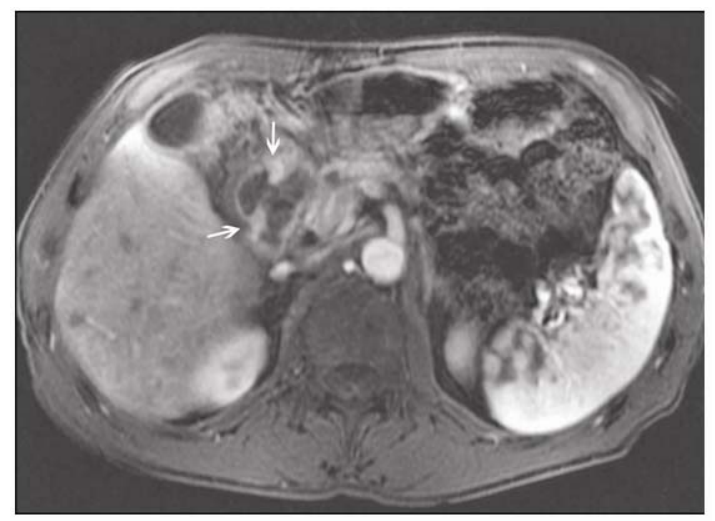


The role of MRI (and cross-sectional imaging in general) in the diagnosis of PUD is quite limited, although the incidental finding of a mushroom-shaped penetrating ulcer as seen in this case is well worth noting by virtue of its risk of perforation or bleeding. Differential diagnosis includes duodenal diverticula, which generally have thinner walls and lack a narrow neck, and do not demonstrate mural thickening or enhancement after gadolinium administration.

The duodenum is a rare location for neuroendocrine tumors (NETs) (see Figure 2), representing 2\%-3\% of all gastrointestinal NETs ${ }^{[6]}$. The majority $(62 \%)$ are G-cell, or gastrin-producing tumors, one third of which are functional tumors that cause clinical manifestations of Zollinger-Ellison syndrome ${ }^{[7]}$. G-cell NETs are strongly associated with multiple endocrine neoplasia syndrome type 1 (MEN-1), and occur in 90\% of MEN-1 patients; however, duodenal G-cell NETs can also occur sporadically ${ }^{[8]}$. Somatostatin-producing (D-cell) tumors account for $21 \%$ of duodenal NETs and occur exclusively around the ampulla of Vater. There is a strong association between D-cell NETs and neurofibromatosis type 1 (NF 1), and the majority of NF-1 patients with D-cell NETs are black women ${ }^{[7]}$.

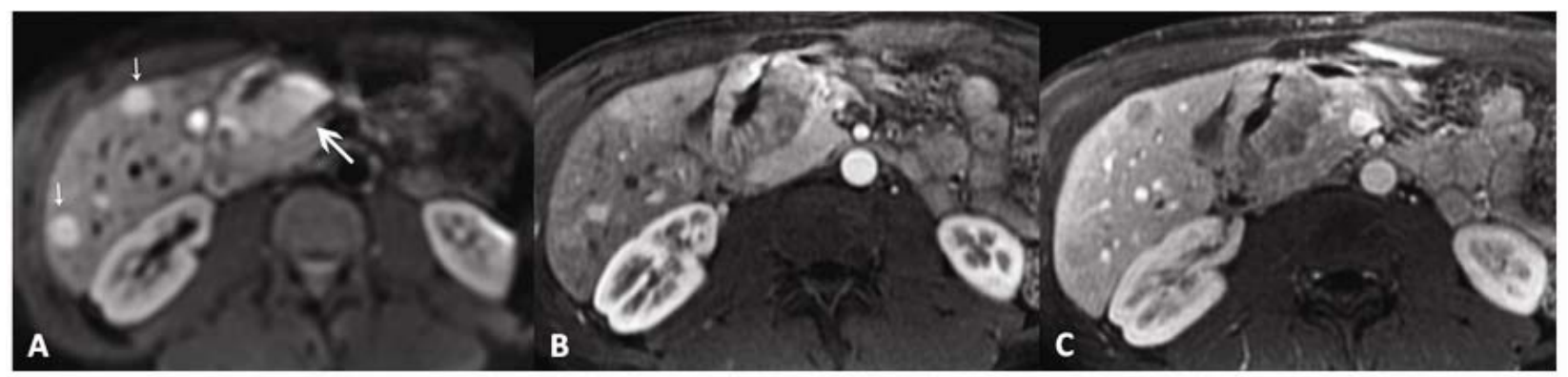

Figure 2. Axial diffusion-weighted image (A) $\left(b=100 \mathrm{~s} / \mathrm{mm}^{2}\right)$ from a 39 year-old man with a history of GI bleeding and thickened duodenum on prior CT demonstrates marked circumferential thickening of the duodenum (large arrow) as well as two small hepatic metastases (small arrows). Axial arterial phase (B) and portal venous phase (C) post-gadolinium 3D SPGR images reveal similar findings. Duodenal NET was found at biopsy.

The MR appearance of duodenal NETs is not well described, and lesions may appear either as a polypoid intraluminal mass or as a mural mass, as seen in this case. Some authors have noted that arterial phase hyperenhancement can help to distinguish these lesions from adenocarcinomas of the duodenum, ampulla, and pancreas, which are typically hypoenhancing ${ }^{[7]}$. The lesion in Figure 2 does not show impressive arterial phase hyperenhancement, although the hyperenhancing hepatic metastases would be atypical for an adenocarcinoma arising in a similar location.

Ampullary adenomas (see Figure 3) are benign but premalignant lesions, which may transform into ampullary carcinomas ${ }^{[9]}$. Ampullary carcinomas (and adenomas) can arise from either of the two types of epithelium found in the region of the ampulla, and are classified as intestinal-type or pancreaticobiliary-type lesions, with some evidence suggesting that the intestinal-type carcinomas might have a more favorable prognosis ${ }^{[10]}$. Most ampullary adenomas $(>90 \%)$ arise from intestinal epithelium ${ }^{[1]}$. On MRI, ampullary adenomas typically appear as small nodular lesions projecting from the ampulla into the duodenal lumen with heterogeneous increased T2 signal intensity and mild-moderate enhancement after gadolinium administration. Ampullary adenomas, by virtue of their location, frequently cause obstruction of the biliary and pancreatic ducts.

Visualization of an ampullary adenoma or carcinoma with MRI can be very difficult, particularly with a small, non-papillary mass, and serendipitous diagnosis will likely occur only for relatively large lesions. Distention of the duodenum with oral contrast, high spatial resolution acquisitions, and the liberal use of SSFP and SSFSE sequences (minimizing motion artifact from peristalsis) might help to improve the sensitivity for detection of small masses. 
Distinguishing between an ampullary adenoma and carcinoma can be difficult, with larger size, infiltrative appearance, and metastatic disease all suggesting a malignant lesion. Differential diagnosis includes ampullary stenosis, pancreatic adenocarcinoma, distal cholangiocarcinoma, and duodenal carcinoma.
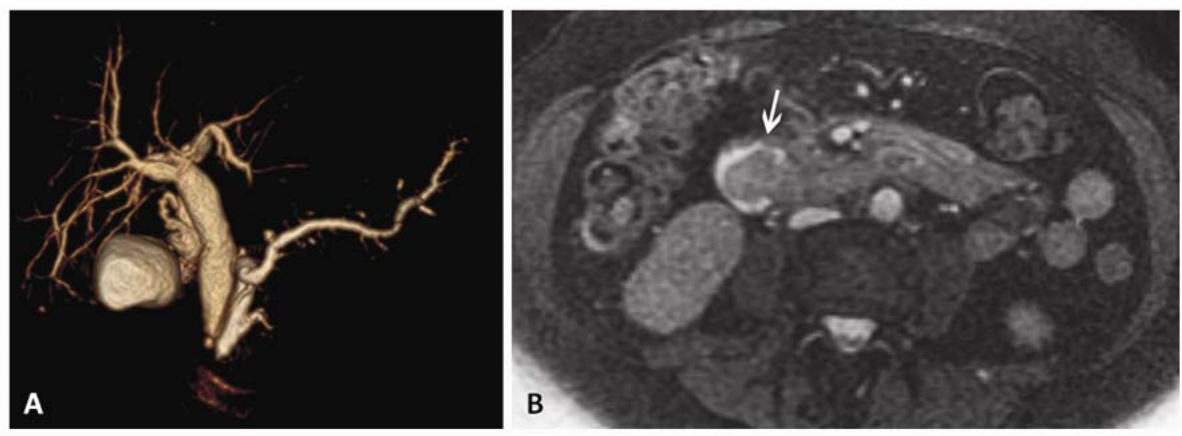

Figure 3. Volume-rendered image from 3D FSE MRCP (A) in a 75 year-old woman with a history of lymphoma shows diffuse dilatation of biliary and pancreatic ducts, with obstruction occurring at the level of the ampulla. Axial fatsuppressed b-SSFP image (B) demonstrates a polypoid mass projecting from the ampulla into the duodenum (arrow). Biopsy revealed ampullary adenoma.

The duodenum is an unusual location for primary extranodal lymphoma (see Figure 4). Primary gastrointestinal lymphoma accounts for $4 \%-20 \%$ of all non-Hodgkin's lymphoma and $25 \%-40 \%$ of all extranodal lymphoma. Most gastrointestinal (GI) tract lymphoma (80\%-90\%) occurs in the stomach and small bowel. The duodenum is the least common site, accounting for $1 \%-2 \%$ of lesions ${ }^{[12]}$.

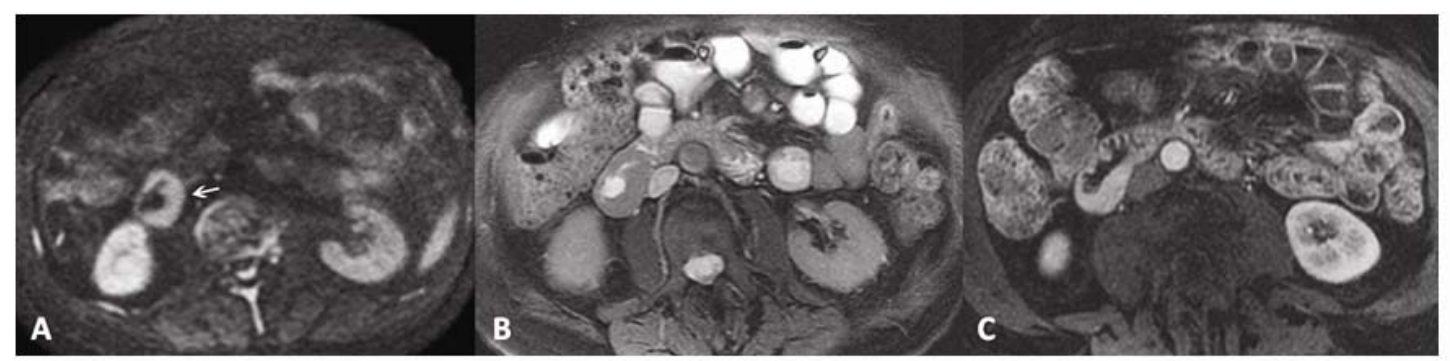

Figure 4. Axial diffusion-weighted image $(\mathrm{A})\left(\mathrm{b}=600 \mathrm{~s} / \mathrm{mm}^{2}\right)$ in a 66 year-old man with abdominal pain and GI bleeding reveals moderate mural thickening and increased signal intensity involving the $2^{\text {nd }}$ portion of the duodenum (arrow). Axial fat-suppressed b-SSFP image (B) shows similar findings, and the lesion demonstrates moderate diffuse hyperenhancement on a portal venous phase post gadolinium 3D SPGR image (C). Biopsy revealed plasmablastic lymphoma.

There are few reports of duodenal lymphoma in the MR literature; however, descriptions of the appearance on CT have emphasized focal or diffuse fold thickening, often in association with adjacent lymphadenopathy ${ }^{[13]}$. This case demonstrates smooth focal fold thickening with moderately increased signal intensity on diffusion-weighted images and moderate enhancement following gadolinium administration. Differential diagnosis includes duodenal adenocarcinoma and NET as well as metastatic disease.

\subsection{Small bowel lesions}

Ectopic pancreas (see Figure 5) is defined as pancreatic tissue lacking anatomic and vascular continuity with the rest of the gland, and is most often located in the stomach, duodenum, or jejunum. Most lesions are small and asymptomatic and are discovered incidentally at endoscopy, surgery, or cross sectional imaging. Lesions may become symptomatic if pancreatitis or a pancreatic tumor develops within the ectopic tissue. 
Ectopic pancreas typically appears as an enhancing submucosal mass in the stomach or duodenum, for which the differential diagnosis includes gastrointestinal stromal tumor (GIST), NET, and leiomyoma. A recent MRI study ${ }^{[14]}$ evaluating findings in patients with ectopic pancreas and those with other submucosal tumors found high sensitivity and specificity in comparing the signal intensity of the lesion with native pancreas. Ectopic pancreas followed the signal intensity of native pancreas, while other submucosal lesions tended to show higher T2 signal intensity and lower T1 signal intensity. The authors found the pre-gadolinium T1-weighted images particularly helpful, where ectopic pancreas appeared relatively bright, as illustrated by Figure 5A.

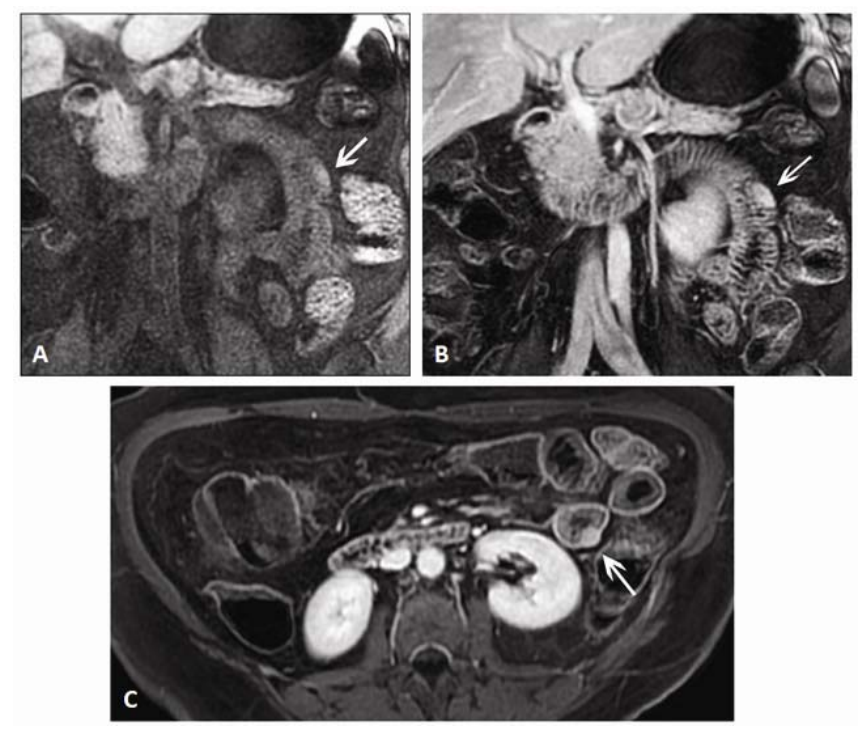

Figure 5. Coronal pre-gadolinium (A), and coronal and axial post-gadolinium (B-C) 3D SPGR images in a 54 year-old woman with suspected partial small bowel obstruction demonstrate a small ovoid hyperenhancing mass (arrows) in the lateral wall of the proximal jejunum. Note that this lesion is relatively hyperintense on the pre-contrast image, similar in signal intensity to the adjacent pancreas. Biopsy revealed ectopic pancreatic tissue.

GISTs (see Figure 6) are cellular spindle cell, epithelioid, pleomorphic mesenchymal tumors that express the Kit protein a stem cell factor receptor protein whose activation leads to unchecked cell growth and resistance to apoptosis ${ }^{[15]}$. GISTs are relatively rare tumors, accounting for $1 \%-3 \%$ of all GI neoplasms, but are nevertheless the most common GI mesenchymal tumor. The peak age of incidence is 50-60 years, with a slight male predominance. Most tumors arise in the stomach (60\%-70\%) or small intestine (20\%-30\%), with additional lesions occurring less frequently in the esophagus, colon, rectum, and mesentery. Approximately $20 \%-30 \%$ of these lesions are malignant.

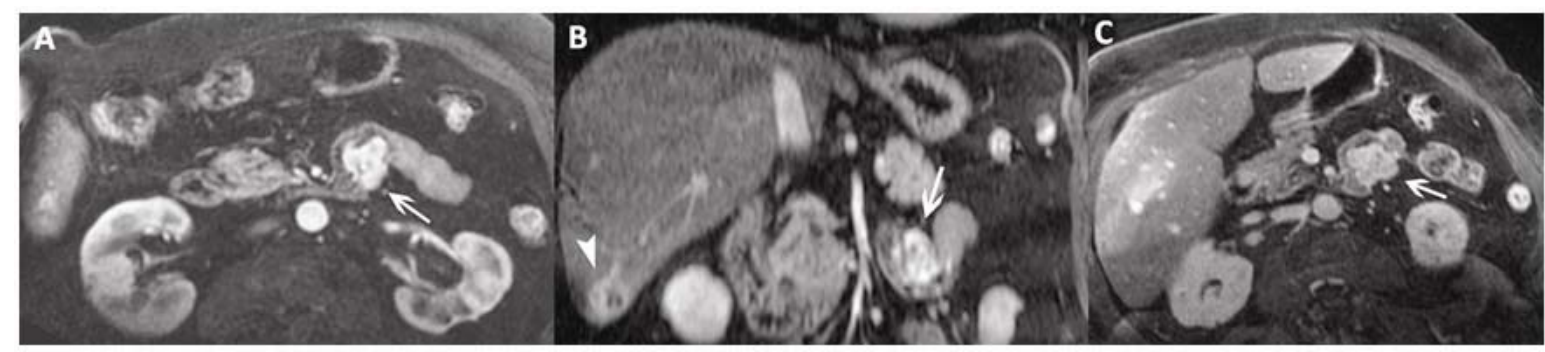

Figure 6. Axial (A) and coronal oblique reformatted (B) arterial phase post-gadolinium 3D SPGR images from a 73 year-old woman with a hepatic mass found at sonography demonstrate a lobulated, exophytic hyperenhancing mass (arrows) projecting from the wall of the proximal jejunum. Axial oblique reformatted venous phase image (C) shows slight washout and faint rim enhancement of the jejunal mass. Best seen on (B) is a hyperenhancing metastasis in the inferior right hepatic lobe (arrowhead). Biopsy revealed GIST. 
The appearance of GISTs on MRI has not been widely documented in the literature ${ }^{[16,17]}$. Most lesions originate in the muscularis propria of the intestinal wall, and therefore often exhibit an exophytic growth pattern, although intraluminal polypoid lesions can occasionally be seen. Smaller lesions, as illustrated in Figure 6, are more often uniform, showing mildly-moderately increased signal intensity on T2-weighted images and uniform hyperenhancement on arterial phase post gadolinium images. The larger lesions are more often heterogeneous, with multiple regions of cystic degeneration or necrosis.

Peutz-Jeghers syndrome (PJS) (see Figure 7) is a familial polyposis syndrome with autosomal dominant inheritance and is often associated with mutation in the STK11 gene. PJS is characterized by mucocutaneous pigmented lesions as well as by multiple hamartomatous polyps throughout the GI tract ${ }^{[18]}$. Small bowel polyps arise at an early age and may cause anemia, bleeding, obstruction, or intussusception. The lifetime incidence of malignancy in PJS patients is markedly increased ( $93 \%$ by the age of 65 years), with the small bowel most commonly affected ${ }^{[19]}$. A few authors have evaluated MRE as a surveillance technique in PJS ${ }^{[20,21]}$, with varying degrees of success. MRE is unlikely to detect very small polyps; however larger lesions should be visible, and complications such as obstruction or intussusception, as in this example, can be easily detected.

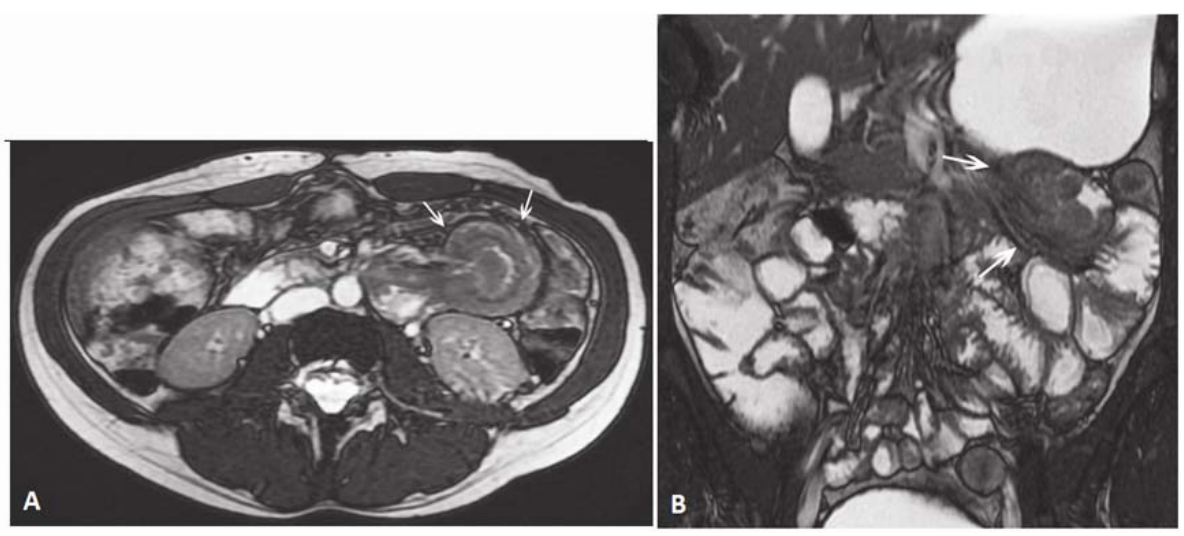

Figure 7. Axial (A) and coronal (B) b-SSFP images from 30 year-old woman with Peutz-Jeghers syndrome and intermittent abdominal pain demonstrate intussusception of the jejunum associated with multiple polypoid intraluminal lesions (arrows).

Lipomatous polyposis (see Figure 8) is a rare condition consisting of multiple submucosal (or less commonly subserosal) lipomas which can occur throughout the GI tract ${ }^{[22]}$. In this example the lesions involve stomach and small bowel, sparing the colon. Histologically the lesions comprise submucosal collections of mature adipocytes often surrounded by a fibrous capsule. While this condition can be asymptomatic, patients may present with symptoms of gastrointestinal bleeding, diarrhea, and intussusception or obstruction. Lipomatous polyposis has been associated with two rare syndromes: familial multiple lipomatosis and Weber-Christian disease (febrile relapsing nonsuppurative panniculitis). Keys to the diagnosis include identification of the multiple gastric and small intestinal polyps, and recognition that they are composed of fat.

Intestinal lymphangiectasia (see Figure 9) is a rare protein-losing enteropathy characterized by dilatation of mucosal and submucosal lymphatic vessels in small bowel, which in turn results in mural and fold thickening, predominantly involving the jejunum ${ }^{[23,24]}$. Intestinal leakage of plasma proteins occurs through elevated lymphatic pressure, which can be a result of a primary lymphatic dysplasia (thought to be the cause in this patient) or a secondary effect of various diseases, including constrictive pericarditis, Whipple's disease, lymphoma, sarcoidosis, and Crohn's disease. Clinical signs and symptoms include peripheral edema, diarrhea, steatorrhea, ascites, and pleural effusions. Differential diagnosis for small bowel fold thickening includes Whipple's disease and lymphoma, which are often accompanied by adenopathy and splenomegaly, as well as amyloidosis, and eosinophilic gastroenteritis. 


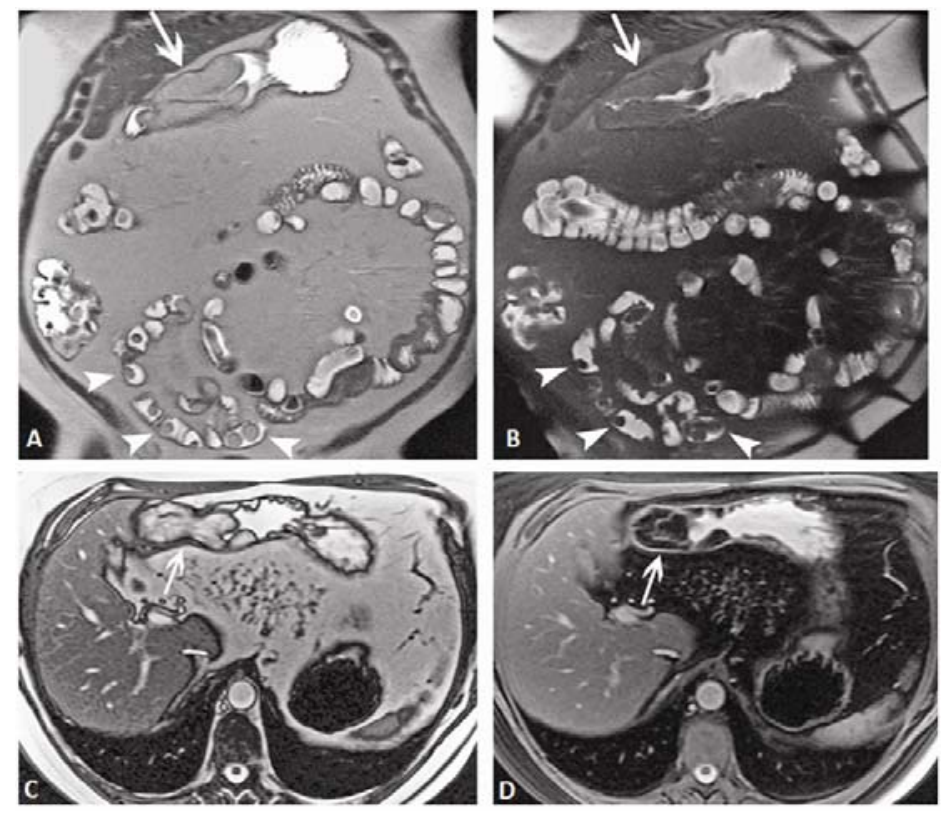

Figure 8. Coronal SSFSE images without (A) and with (B) fat suppression in a 47 year-old man with lipomatous polyposis reveal marked nodular thickening of the gastric antrum (arrows) as well as several small polypoid lesions in the ileum (arrowheads), all of which show signal loss on the fat-suppressed image. Axial b-SSFP images without (C) and with (D) fat suppression in the upper and lower abdomen show similar findings in the stomach (arrows). Note also marked diffuse fatty infiltration of the pancreas.

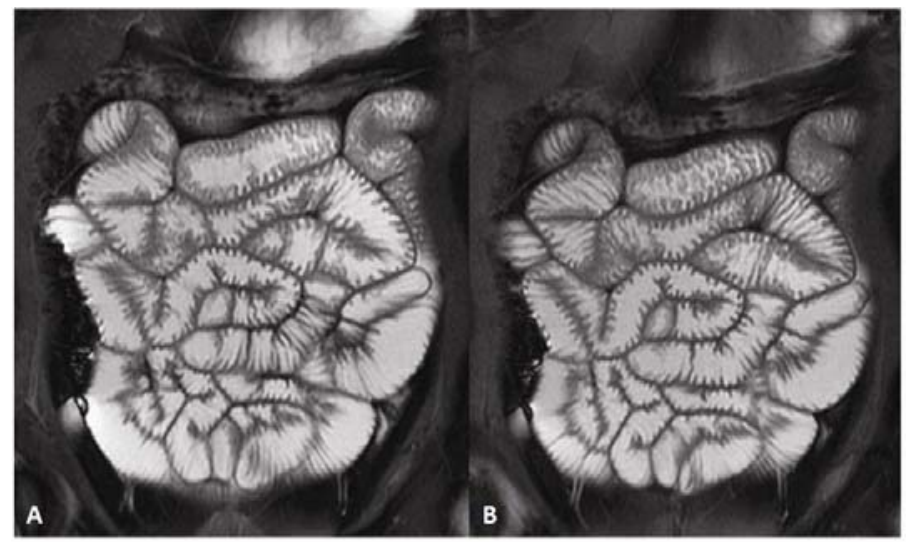

Figure 9. Coronal fat-suppressed b-SSFP images (A-B) in a 22 year-old woman with protein losing enteropathy and intestinal lymphangiectasia reveal diffuse fold thickening in jejunal loops.

\subsection{Mesenteric lesions}

Mesenteric venous thrombosis (see Figure 10) accounts for 5\%-15\% of all mesenteric ischemic events, and usually involves the superior mesenteric vein. The presentation of mesenteric venous thrombosis can be acute, as in this case, or subacute or chronic. Acute thrombosis carries a significant risk of bowel infarction and peritonitis, and reported mortality rates range from $20 \%-50 \%{ }^{[25]}$. Most frequent etiologies associated with mesenteric vein thrombosis include coagulopathies, cancer, inflammatory conditions, postoperative state, and portal hypertension, with roughly $25 \%$ of cases idiopathic ${ }^{[25]}$. The appearance of infarcted bowel on MRI can be nonspecific, demonstrating mural thickening and adjacent fluid and/or inflammation, for which the differential diagnosis includes inflammatory and infectious etiologies; however the expansile thrombus in the superior mesenteric vein in this case (see Figure 10a) makes the diagnosis relatively straightforward. 


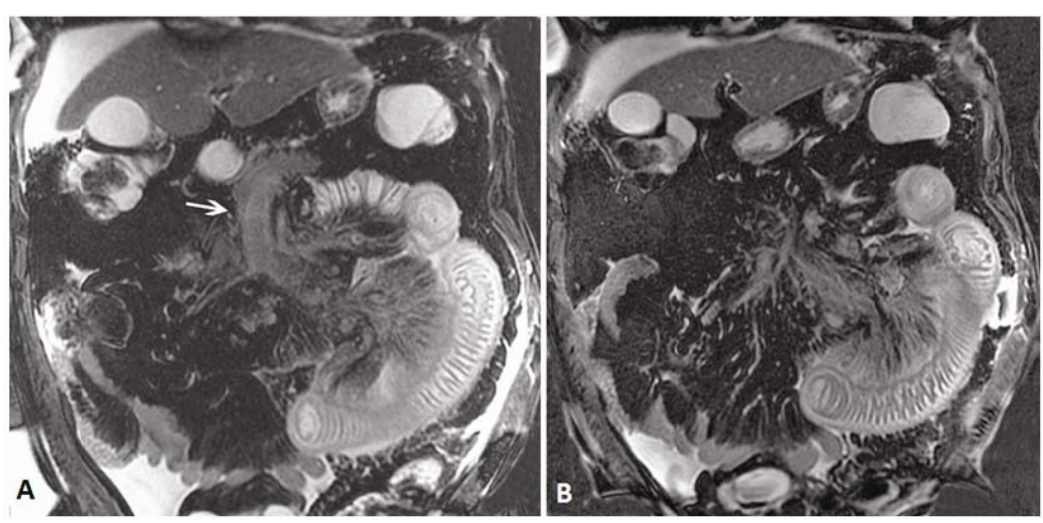

Figure 10. Coronal fat-suppressed b-SSFP images (A-B) in a 53 year-old man with sudden onset of severe epigastric pain reveal marked mural thickening of a jejunal loop with adjacent inflammatory changes in the mesentery and abdominal ascites. Note also hypointense thrombus expanding the superior mesenteric vein (arrow in A). Surgery revealed jejunal necrosis.

Inhibitors of tumor necrosis factor $\alpha$ (TNF- $\alpha$ ) are effective for management of rheumatic and autoimmune disorders, and have been increasingly used in patients with disease resistant to more conventional therapies. Along with their increasing application, however, have come a few reports of paradoxical secondary autoimmune conditions, such as vasculitis (see Figure 11). A recent paper, for example, has documented vasculitis in eight patients associated with anti-TNF- $\alpha$ therapy ${ }^{[26]}$.
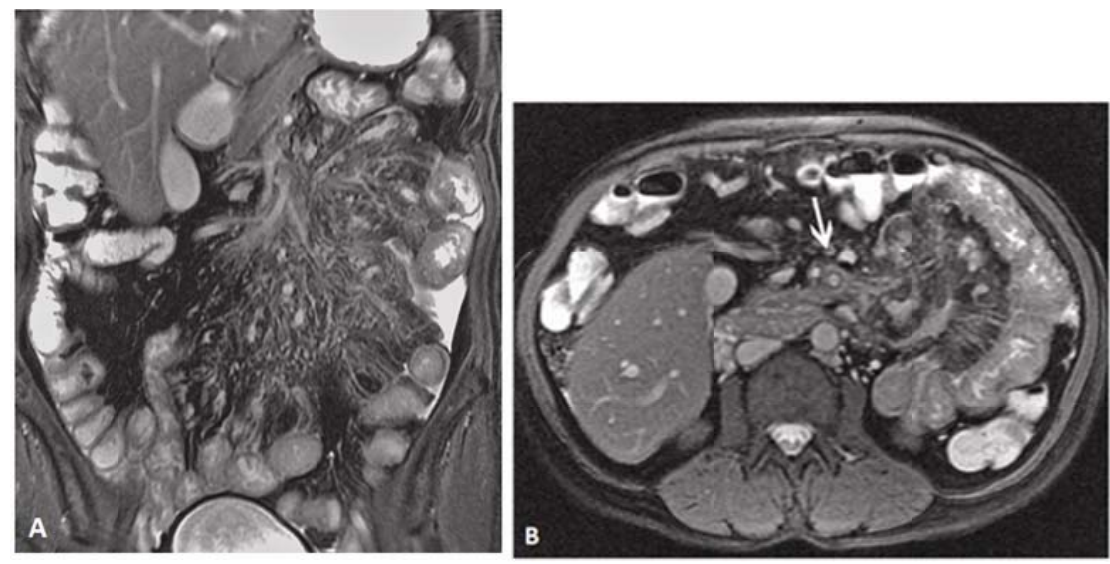

Figure 11. Coronal (A) and axial (B) fat-suppressed b-SSFP images from a 56 year-old woman with a long history of Crohn's disease demonstrate marked thickening of a jejunal loop in the mid abdomen with small amount of adjacent ascites. Note also diffuse mesenteric stranding and multiple small mesenteric lymph nodes as well as soft tissue encasement of the superior mesenteric artery best seen on the axial image (arrow). Findings are consistent with vasculitis related to anti-TNF- $\alpha$ therapy.

The focal thickening of a jejunal loop as well as the prominence of the adjacent mesenteric vessels illustrated in Figure 11 in a patient with a history of Crohn's disease receiving anti-TNF- $\alpha$ therapy could be seen in inflammatory bowel disease, although the ileum is more commonly affected. The extensive mesenteric inflammation, however, would be unusual for uncomplicated IBD, and the extensive circumferential soft tissue encasement of the superior mesenteric artery is typical of vasculitis.

Sclerosing mesenteritis (SM) (see Figure 12) is a rare benign chronic inflammatory process involving the small bowel mesentery. The range of histologic presentation is fairly broad: when inflammation and fat necrosis are the predominant 
components, the term "mesenteric panniculitis" is also used, whereas "retractile mesenteritis" describes a variant of sclerosing mesenteritis where retraction and fibrosis predominate ${ }^{[27,28]}$.
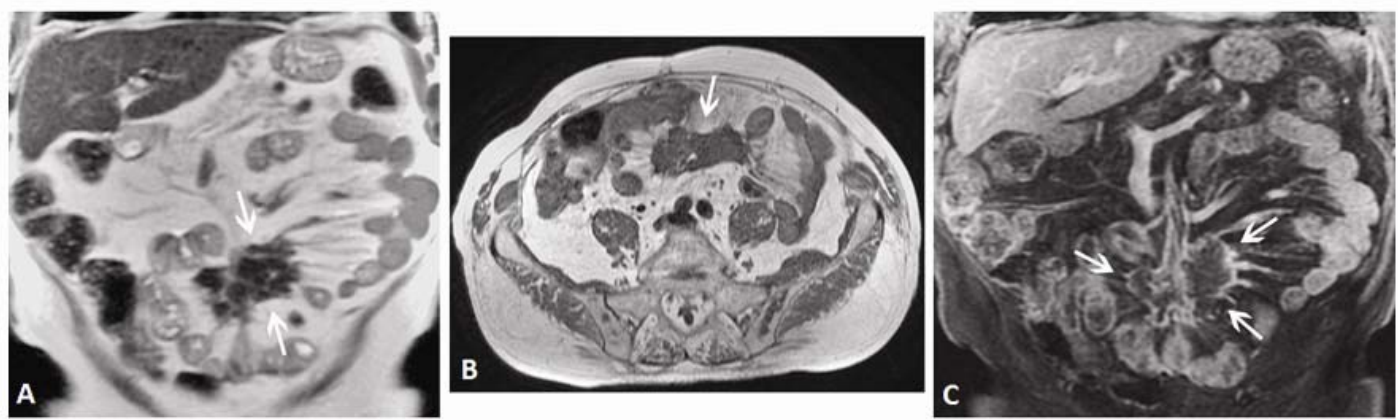

Figure 12. Coronal SSFSE image (A) in an 80 year-old man with known sclerosing mesenteritis demonstrates a low signal intensity mesenteric mass (arrows) with radiating strands of fibrosis and slight tethering of an adjacent small bowel loop. Axial FSE T1-weighted image (B) shows similar findings. Coronal post-gadolinium 3D SPGR image (C) demonstrates slight rim enhancement of the mesenteric mass (arrows).

Imaging features depend on the particular disease variant, but may include inflammatory changes in mesenteric fat. A focal mesenteric mass is often noted in retractile mesenteritis, and is seen in Figure 12, where the low signal intensity on SSFSE images and lack of enhancement following gadolinium administration reflect calcification, a relatively common finding ${ }^{[29]}$. Differential diagnosis includes mesenteric carcinoid, which may have an identical appearance, lymphoma, desmoid tumor, and peritoneal carcinomatosis. The retraction, kinking and tethering of adjacent small bowel loops seen in SM is sometimes characteristic, but may occasionally be difficult to distinguish from carcinoid tumors or from intraabdominal desmoid tumors. An additional distinguishing feature of SM is that, in contrast to desmoid tumors and carcinoid, it does not directly invade adjacent small bowel loops.

Desmoid tumors (see Figure 13) are uncommon mesenchymal neoplasms consisting of elongated spindle cells separated by bands of collagen, which may or may not include myxoid elements. Most desmoids occur in the abdomen or pelvis, either within the mesentery (more common in association with familial adenomatous polyposis) or within the abdominal wall ${ }^{[30,31]}$. The MR appearance of desmoids is somewhat variable: signal intensity on T2-weighted images depends on the relative amounts of cellular tissue, collagen, and myxoid material, but most lesions are mildly hyperintense relative to skeletal muscle. A fairly reliable feature of desmoids (seen in this case) is their increasing enhancement on equilibrium and delayed phase post-gadolinium acquisitions, a characteristic shared by many fibrotic tumors.

There is a strong association between desmoid tumors and familial adenomatous polyposis (FAP). The intraabdominal desmoid tumors seen in FAP patients tend to be smaller and more infiltrative in comparison to sporadically occurring lesions, and are more frequently multiple ${ }^{[32]}$. Sporadically occurring lesions may present as large, mildly heterogeneous masses with relatively well-defined borders, mimicking a large GIST or sarcoma. Infiltration into adjacent small bowel loops can lead to obstruction, fistula formation, or perforation.

Carcinoid tumors (see Figure 14) are well-differentiated neuroendocrine tumors originating from the enterochromaffin cells of the digestive tract or tracheobronchial system. Most lesions occur in the GI tract, and most of these originate from the distal ileum ${ }^{[8]}$. The primary lesions are often small and difficult to detect with CT or MRI, appearing as small enhancing mural nodules, often with associated focal mural thickening. Frequently initial diagnosis is made by visualizing mesenteric extension or metastasis: the release of vasoactive substances such as serotonin can generate an intense desmoplastic reaction, leading to the characteristic kinking, retraction, and angulation of adjacent small bowel loops. Encasement of mesenteric arteries and veins is frequent, and may lead to intestinal ischemia ${ }^{[8,33,34]}$. 
The major differential diagnosis for carcinoid with mesenteric involvement is SM. Evidence of a primary small bowel lesion or metastatic disease to the liver or omentum favors carcinoid. Small bowel adenocarcinoma can also have a similar appearance, although the angulation and tethering of small bowel loops is less prominent, and more often the appearance is dominated by an annular constricting mural mass. A majority of patients with small bowel carcinoid have metastatic disease at the time of diagnosis, although survival rates compare favorably with most other metastatic tumors.

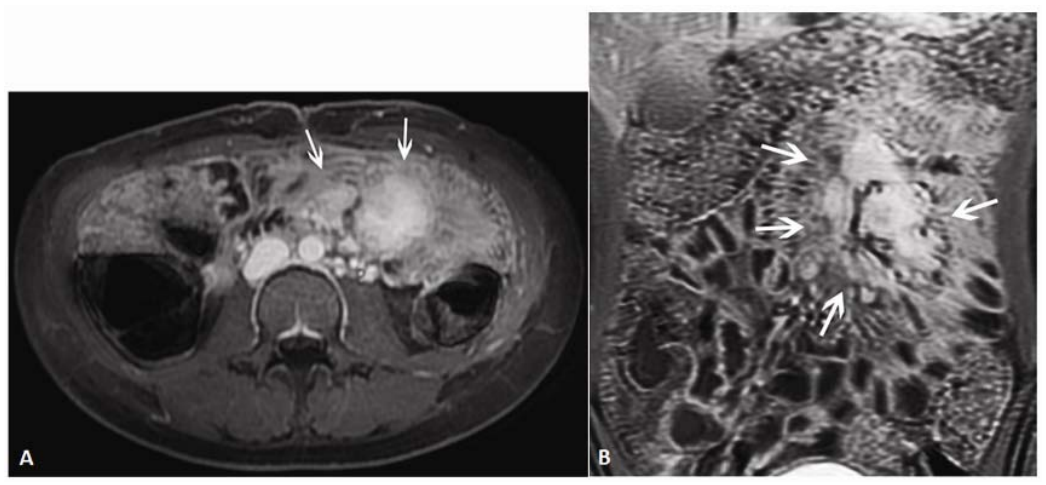

Figure 13. Axial portal venous phase post-gadolinium 3D SPGR image (A) from a 34 year-old woman with familial adenomatous polyposis reveals mildly hyperintense ill-defined masses in the mesenteric root (arrows). 5 minute delayed coronal 3D SPGR image (B) demonstrates increasing hyperenhancement of the lesions (arrows), poorly-defined margins, and encasement of the superior mesenteric artery. Surgical pathology revealed desmoid tumors.

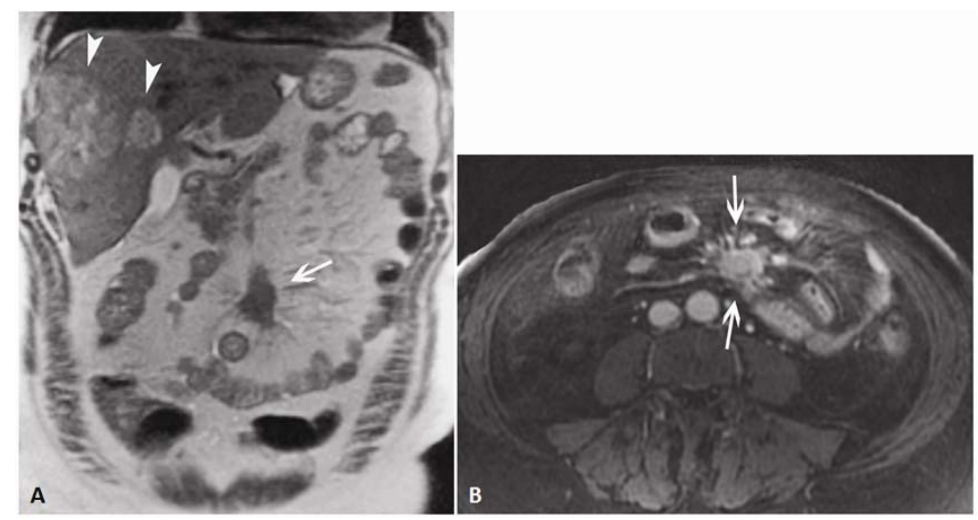

Figure 14. Coronal SSFSE image (A) from a 63 year-old man with intermittent abdominal pain and diarrhea demonstrates a spiculated mass at the root of the mesentery (arrows) as well as large hepatic metastases (arrowheads). Axial post-gadolinium 3D SPGR image (B) shows a spiculated mildly enhancing mesenteric mass (arrows) encasing the distal superior mesenteric artery. Surgical pathology revealed carcinoid tumor. Small primary tumors were identified at surgery in the distal ileum and could not be seen on the MRI examination.

\subsection{Limitations of MRI and comparison with other imaging techniques}

Both MRI and CT allow detection and characterization of mural and endoluminal small bowel lesions, particularly when enterography techniques are employed. MRI excels in lesion characterization, and the appearance of an abnormality on multiple different pulse sequences can sometimes lead to a definitive diagnosis. On the other hand, acquisition times both for individual series as well as for the entire exam are long in comparison to CT, leading to challenges with motion artifact and patient cooperation. A major limitation of $\mathrm{CT}$ in comparison to MRI is its use of ionizing radiation, which is especially problematic in young patients with IBD who require multiple examinations throughout their lives. Sonography is increasingly employed in assessment of small bowel, particularly in the pediatric population, and has shown to be fairly accurate in identifying diseased segments of bowel (in IBD, for example). Sonography is more operator-dependent than 
other cross-sectional modalities, segments of bowel are more often inaccessible or obscured, and comprehensive survey of the entire small bowel is difficult. PET-CT is commonly used as a problem-solving technique for staging known bowel neoplasms, and has greater sensitivity and specificity for identification of extrahepatic metastatic disease than CT or MRI. The low spatial resolution of PET, its requirement for ionizing radiation, and occasional overlap in the appearance of inflammatory and neoplastic etiologies are all limitations. Endoscopy remains the gold standard for visualization of small bowel lesions, with the opportunity for immediate biopsy when a mass is identified. Endoscopy is an invasive technique, and surveillance of the entire small bowel is problematic. Endoscopy also has limited ability to detect serosal and mesenteric lesions, and limited scope in staging neoplasms.

\section{Conclusions}

An increasing number and variety of unusual lesions of the small bowel and mesentery will likely be encountered as MR enterography becomes a widely accepted and popular technique. Standard MRE protocols are often effective for characterization of these lesions, and may allow a relatively narrow differential diagnosis. Abnormalities of small bowel and mesentery are also occasionally encountered during standard abdominal MRI examinations, where the opportunity to perform additional acquisitions tailored to the particular lesion may be helpful for complete and timely characterization.

\section{References}

[1] Lee SS, Kim AY, Yang SK, et al. Crohn disease of the small bowel: comparison of CT enterography, MR enterography, and small-bowel follow-through as diagnostic techniques. Radiology. 2009; 25: 751-761. PMid:19276325 http://dx.doi.org/10.1148/radiol.2513081184

[2] Hafeez R, Punwani S, Boulos P, et al. Diagnostic and therapeutic impact of MR enterography in Crohn's disease. Clin Radiol. 2011; 66: 1148-58. PMid:21943719 http://dx.doi.org/10.1016/j.crad.2010.12.018

[3] Fiorino G, Bonifacio C, Peyrin-Biroulet L, et al. Prospective comparison of computed tomography enterography and magnetic resonance enterography for assessment of disease activity and complications in ileocolonic Crohn's disease. Inflamm Bowel Dis. 2011; 17: 1073-80. PMid:21484958 http://dx.doi.org/10.1002/ibd.21533

[4] Huang JQ, Sridhar S, Hunt RH. Role of Helicobacter pylori infection and non-steroidal anti-inflammatory drugs in peptic-ulcer disease: a meta-analysis. Lancet. 2002; 359: 14-22. http://dx.doi.org/10.1016/S0140-6736(02)07273-2

[5] Feinstein LB, Holman RC, Yorita Christensen KL, et al. Trends in hospitalization for peptic ulcer disease, United States, 1998 2005. Emerg Infect Dis. 2010; 16: 1410-18. PMid:20735925 http://dx.doi.org/10.3201/eid1609.091126

[6] Modlin IM, Lye KD, Kidd M. A 5-decade analysis of 13,715 carcinoid tumors. Cancer. 2003; 97: 934-59. PMid:12569593 http://dx.doi.org/10.1002/cncr.11105

[7] Levy AD, Taylor LD, Abbott RM, et al. Duodenal carcinoids: imaging features with clinical-pathologic comparison. Radiology 2005; 237: 967-72. PMid:16237144 http://dx.doi.org/10.1148/radiol.2373041863

[8] Levy AD, Sobin LH. Gastrointestinal carcinoids: imaging features with clinicopathologic comparison. Radiographics. 2007; 27: 237-57. PMid:17235010 http://dx.doi.org/10.1148/rg.271065169

[9] Nikolaidis P, Hammond NA, Day K, et al. Imaging features of benign and malignant ampullary and periampullary lesions. Radiographics. 2014; 34: 624-41. PMid:24819785 http://dx.doi.org/10.1148/rg.343125191

[10] Chung YE, Kim MJ, Park MS, et al. Differential features of pancreatobiliary- and intestinal-type ampullary carcinomas at MR imaging. Radiology. 2010; 257: 384-93. PMid:20829529 http://dx.doi.org/10.1148/radiol.10100200

[11] Albores-Saavedra J, Henson DE, Klimstra DS. Tumors of the gallbladder, extrahepatic bile ducts, and ampulla of Vater. In: AFIP atlas of tumor pathology, series III. Washington, DC: Armed Forces Institute of Pathology, 2000.

[12] Chestovich PJ, Schiller G, Sasu S, et al. Duodenal lymphoma: a rare and morbid tumor. American Surgeon. 2007 ; 73: $1057-62$. PMid:17983081

[13] Wei CJ, Chiang JH, Lin WC, et al. Tumor and tumor-like lesions of duodenum: CT and barium imaging features. J Clin Im. 2003; 27: 89-96. http://dx.doi.org/10.1016/S0899-7071(02)00494-1

[14] Jang KM, Kim SH, Park HJ, et al. Ectopic pancreas in upper gastrointestinal tract: MRI findings with emphasis on differentiation from submucosal tumor. Acta Radiol. 2013; 54: 1107-16. PMid:23858505 http://dx.doi.org/10.1177/0284185113491251 
[15] Levy AD, Remotti HE, Thompson WM, et al. Gastrointestinal stromal tumors: radiologic features with pathologic correlation. Radiographics. 2003; 23: 283-304. PMid:12640147 http://dx.doi.org/10.1148/rg.232025146

[16] Darnell A, Dalmau E, Pericay C, et al. Gastrointestinal stromal tumors. Abdom Imaging. 2006; 31: 387-399. PMid:16465584 http://dx.doi.org/10.1007/s00261-004-0092-8

[17] Chourmouzi D, Sinakos E, Papalavrentios L, et al. Gastrointestinal stromal tumors: a pictorial review. J Gastrointestin Liver Dis. 2009; 18: 379-83. PMid:19795038

[18] Kopacova M, Tacheci I, Rejchrt S, et al. Peutz-Jeghers syndrome: diagnostic and therapeutic approach. World J Gastroenterol. 2009; 15: 5397-408. PMid:19916169 http://dx.doi.org/10.3748/wjg.15.5397

[19] Lim W, Olschwang S, Keller JJ, et al. Relative frequency and morphology of cancers in STK11 mutation carriers. Gastroenterology. 2004; 126: 1788-94. PMid:15188174 http://dx.doi.org/10.1053/j.gastro.2004.03.014

[20] Maccioni F, Ansari NA, Mazzamurro F, et al. Surveillance of patients affected by Peutz-Jeghers syndrome: diagnostic value of MR enterography in prone and supine position. Abdom Imaging. 2012; 37: 279-87. PMid:21538021 http://dx.doi.org/10.1007/s00261-011-9739-4

[21] Gupta A, Postgate AJ, Burling D, et al. A prospective study of MR enterography versus capsule endoscopy for the surveillance of adult patients with Peutz-Jeghers syndrome. AJR. 2010; 195: 108-16. PMid:20566803 http://dx.doi.org/10.2214/AJR.09.3174

[22] Ward EM, Wolfsen HC. Review article: the non-inherited gastrointestinal polyposis syndromes. Aliment Pharmacol Ther. 2002; 16: 333-42. http://dx.doi.org/10.1046/j.1365-2036.2002.01172.x

[23] Fakhri A, Fishman EK, Jones B, et al. Primary intestinal lymphangiectasia: clinical and CT findings. J Comput Assist Tomogr. 1985; 9: 767-770. PMid:4019833

[24] Holzknecht N, Helmberger T, Beuers U, et al. Cross-sectional imaging findings in congenital intestinal lymphangiectasia. J Comput Assist Tomogr. 2002; 26: 526-8. PMid:12218814 http://dx.doi.org/10.1097/00004728-200207000-00009

[25] Kumar S, Sarr KG, Kamath PS. Mesenteric venous thrombosis. N Engl J Med. 2001; 345: 1683-88. PMid:11759648 http://dx.doi.org/10.1056/NEJMra010076

[26] Sokumbi O, Wetter DA, Makol A, et al. Vasculitis associated with tumor necrosis factor-alpha inhibitors. Mayo Clin Proc. 2012; 87: 739-45. PMid:22795634 http://dx.doi.org/10.1016/j.mayocp.2012.04.011

[27] Levy AD, Rimola J, Mehrotra AK, et al. Benign fibrous tumors and tumorlike lesions of the mesentery: radiologic-pathologic correlation. Radiographics. 2006; 26: 245-64. PMid:16418255 http://dx.doi.org/10.1148/rg.261055151

[28] Vlachos K, Archontovasilis F, Falidas E, et al. Sclerosing mesenteritis: diverse clinical presentations and dissimilar treatment options. A case series and review of the literature. Intl Arch Med. 2011; 4: 17-25. PMid:21635777 http://dx.doi.org/10.1186/1755-7682-4-17

[29] Grieser C, Denecke T, Langrehr, et al. Sclerosing mesenteritis as a rare cause of upper abdominal pain and digestive disorders. Acta Radiol 2008; 49: 744-6. PMid:19143059 http://dx.doi.org/10.1080/02841850802093887

[30] Escobar C, Munker R, Thomas JO, et al. Update on desmoid tumors. Ann Oncol. 2012; 23: 562-69. PMid:21859899 http://dx.doi.org/10.1093/annonc/mdr386

[31] Shinagare AB, Ramaiya NH, Jagannathan JP, et al. A to Z of desmoid tumors. AJR. 2011; 197: W1008-14. PMid:22109314 http://dx.doi.org/10.2214/AJR.11.6657

[32] Healy JC, Reznek RH, Clark SK, et al. MR appearances of desmoid tumors in familial adenomatous polyposis. AJR. 1997; 169: 465-72. PMid:9242755 http://dx.doi.org/10.2214/ajr.169.2.9242755

[33] Bader TR, Semelka RC, Chiu VCY, et al. MRI of carcinoid tumors: spectrum of appearances in the gastrointestinal tract and liver. J Magn Reson Im. 2001; 14: 261-9. PMid:11536403 http://dx.doi.org/10.1002/jmri.1182

[34] Winant AJ, Vora A, Ginter PS, et al. More than just metastases: a practical approach to solid mesenteric masses. Abd Imaging. 2014; 39: 605-21. PMid:24509899 http://dx.doi.org/10.1007/s00261-014-0090-4 